\title{
Analysis of short-term heart rate variability in patients with Crohn's disease
}

\author{
Bei-bei WANG ${ }^{1,2}$, Can CUI ${ }^{2}$, Shan-ying $\mathrm{LIAO}^{2}$, Xiao-ling BU², Wei-hong $\mathrm{SHA}^{1,2 *}$ (D)
}

\begin{abstract}
To investigate the characteristics of heart rate variability in patients with Crohn's disease (CD) and the relationship between HRV parameters and inflammatory activity. Background: CD is a chronic inflammatory granuloma disease of the digestive tract. A total of 37 patients with CD and 39 age- and gender-matched healthy controls were enrolled. Demographic information, medication, and medical history were collected from questionnaires completed by all participants. HRV parameters was recorded by $5 \mathrm{~min}$ short-term heart rate variability analyzer. Inflammatory factors, Crohn's disease activity index (CDAI), and anxiety and depression were assessed. The mean autonomic nerve function score was 72 in CD group, significantly lower than that in control group $(75, \mathrm{P}<0.05)$. The equilibrium in $\mathrm{CD}$ group was strongly lower than that in control group $(\mathrm{P}=0.001)$. $\mathrm{CD}$ subjects had lower autonomic nervous function compared with controls, which positively correlated with body mass index. Equilibrium was significantly lower among CD subjects, which negatively correlated with inflammatory markers $\mathrm{C}$ reactive protein and platelet count. Autonomic dysfunction was observed in CD subjects, mainly manifested as decreased equilibrium, which this inherent imbalance of autonomic function associated with active inflammation.
\end{abstract}

Keywords: autonomic nervous system; autonomic dysfunction; Crohn's disease; gastroenterology and hepatology; heart rate variability.

Practical Application: Analysis of short-term heart rate variability in patients with Crohn's disease.

\section{Introduction}

Crohn's disease $(\mathrm{CD})$ is a chronic inflammatory granuloma disease of the digestive tract, which can be affected through the whole digestive tract from mouth to anus. The intestinal abnormal immune response is considered to be an important factor in CD pathogenesis and chronicization. The autonomic nervous system (ANS) has the function of regulating gastrointestinal movement and secretion. There is increasing evidence on the association between ANS and inflammation in CD patients. And evidence indicates that neurotransmitters can regulate the production of inflammatory cytokines (Boissé et al., 2009).

Heart rate variability (HRV), a quantitative measurement of independent neural activity, can not only provide information about the autonomic nervous system and stress state, but also be measured noninvasively and repeatedly. It has been widely used for clinical evaluation of autonomic neurofunctional disorders in inflammatory diseases and functional gastrointestinal diseases (Malik, 1996; Kleiger et al., 2005; Hill \& Siebenbrock, 2009).

However, the results of studies on autonomic neural function in CD patients lack consistency (Pellissier et al., 2010; Mouzas et al., 2002; Ganguli et al., 2007). No study has been conducted in Chinese population. Furthermore, there is no prospective study linking the association between the abnormality of autonomic neural function and the severity of inflammation or other inflammatory indicators that reflect $\mathrm{CD}$ disease activity. Therefore, through comparing with the healthy control group, the study observed the changes of HRV parameters, explored the possible autonomic neural disorders, and further analyzed the correlation between their changes and inflammatory activity in $\mathrm{CD}$ patients.

\section{Materials and methods}

\subsection{Patients}

The study subjects were recruited CD patients who were admitted to our hospital from January 1, 2018 to December 31,2018 . The inclusion criteria was as followings: (1) patients diagnosed with CD according to 2012 Consensus Opinion on the Diagnosis and Treatment of Inflammatory Bowel Disease (Group CMADMIBD, 2012); (2) over 18 years of age. Exclusion criteria included: (1) combined with endocrine dysfunction (including thyroid dysfunction and diabetes) and systemic inflammatory diseases other than CD; (2) current use of drugs affecting cardiovascular system and conduction, including all anti-arrhythmia drugs, receptor blockers, calcium channel blockers, and sympathetic neurodrugs; (3) known to have neuropathy or other associated diseases; (4) known to have heart diseases such as congenital diseases, valve dysfunction, conduction dysfunction, ischemic heart disease or any degree of congestive heart failure. This study was approved by our ethics committee, and all participants signed an informed consent form before joining the group.

${ }^{1}$ The Second School of Clinical Medicine, Southern Medical University, Guangdong Province, China

${ }^{2}$ Department of Gastroenterology and Hepatology, Guangdong Provincial People's Hospital, Guangdong Academy of Medical Sciences, Guangdong Province, China

*Corresponding author: shaweihong@gdph.org.cn 


\subsection{Data collection}

Demographic information, medical history and drug history of all subjects were collected. Blood samples from the CD group were completed to assess inflammatory activity by laboratory examinations such as $\mathrm{C}$ reactive protein (CRP), routine blood test, IL-6, etc. to assess inflammatory status; albumin (ALB) and body mass index (BMI) to assess the nutritional status of CD patients; CDAI score (Crohn's Disease Activity Index) graded according to the number of bowel movements, abdominal pain and body conditions in the past 1 week to evaluate CD clinical activity. Zung's Anxiety Scale (SAS) and Depression Scale (SDS) were used to assess the patient's anxiety and depression. The Patients completed the evaluation according to their own situation in the past week. The total score multiplied by 1.25 were used as the standard score. According to the common results in Chinese population, the standard score is classified with 50 points.

\subsection{HRV measurement}

All subjects were fasting and deprived of water before detection. Heart rate variation analyzer (General Meditech, Inc. (GMI), model DLP6000) was used for electrocardiogram acquisition, collecting 5 min RR period continuously. During the recording process, the subject was required to completely relax, keep awake, regular breathing, do not speak and move, technician carefully observed the situation of each participant to ensure that there was no subjective discomfort and false motion. In-machine software automatically collected and analyzed data, based on the number of time and frequency domain. The following measurements would be obtained: low-frequency power (LF), high-frequency power ( $\mathrm{HF})$, equilibrium, and autonomic nerve function score. LF reflected the dual effects of sympathetic nerve and vagus nerves. While, HF mainly reflected the extent to which the vagus nerve had a leading role. The equilibrium was calculated mainly by LF/HF ratio, which reflects the degree of balance between sympathetic and vagus nerves. Autonomic nerve function score, representing the overall activity of autonomic nervous system, was used to evaluate the regulatory ability of autonomic nervous system. The reference range for equilibrium was $>0.6$, and autonomic nerve function score ranged from 0 to 100 .

\subsection{Statistical analysis}

The normal distributed measurements were represented by the mean value and standard deviation. The comparison between the two groups was independent sample t-test, and the correlation analysis was made using Person analysis. For skewness distributed data, non-paranormal analysis was used to compare the difference between the two groups, and Spearman analysis was used to assess the correlation. The count data was expressed in number and the ratio (\%), and compared with chi-square test. Statistical analysis was made by SPSS 22.0. P value $<0.05$ was considered statistically significant.

\section{Results}

\subsection{Baseline characteristics}

A total of $37 \mathrm{CD}$ patients who met the above inclusion/exclusion criteria were recruited. And 39 healthy populations, who were age and sex-matched with CD groups were included as control group. There were no statistical differences in age and gender distribution between the comparative CD group of general data and the normal control group (mean age of 31, and 31 for each group, female ratio of $30 \%$ and $33 \%$ for CD and control group, respectively: $\mathrm{P}<0.05$ ) (Table 1 ).

\subsection{Comparison of HRV parameters}

Although with no statistical difference, HRV parameters in CD group was faster, compared to control group (Table 1, $\mathrm{P}>0.05)$. The mean autonomic nerve function score was 72 , (58 to 79) in CD group, significantly lower than that in control group $(75$, ranging from 64 to $82, \mathrm{P}<0.05)$. The equilibrium in $\mathrm{CD}$ group was strongly lower than that in control group, with equilibrium of 0.5062 to 0.2447 , and 0.7203 to 0.1603 , respectively $(\mathrm{P}=0.001)$ (Table 1).

\subsection{Correlation analysis of HRV parameters}

The correlation between HRV parameters with inflammation, nutrition indicators and psycho-psychological assessment (anxiety and depression score) were shown in Table 2. The results suggested that the autonomic neural function score and BMI in CD patients were positively correlated $(\mathrm{P}<0.05)$; and equilibrium was negatively correlated with CRP and PLT $(\mathrm{P}<0.05)$ (Figure 1).

\section{Discussion}

In our study, the autonomic neural function in CD group was lower than that in control group, suggesting decreased ability of autonomic nerve to regulate the body function in $\mathrm{CD}$ patients. This is consistent with previous literature reports

Table 1. Baseline characteristic in CD and control groups.

\begin{tabular}{|c|c|c|c|c|}
\hline & \multicolumn{2}{|c|}{$\operatorname{Total}(\mathrm{n}=76)$} & \multirow{2}{*}{$\mathrm{t} / \mathrm{x} 2 / \mathrm{z}$} & \multirow{2}{*}{$\mathrm{p}$} \\
\hline & $\mathrm{CD}$ & Control & & \\
\hline Age & $31.1351 \pm 12.1659$ & $31.4615 \pm 11.4222$ & 0.1206 & 0.9043 \\
\hline $\operatorname{Sex}(M / F)$ & $26 / 11$ & $26 / 13$ & 0.1141 & 0.7355 \\
\hline $\mathrm{HR}$ & $83.8108 \pm 15.9545$ & $78.5385 \pm 8.2233$ & 1.8244 & 0.0721 \\
\hline ANS & $72(58.25 \sim 79)$ & $75(64 \sim 82)$ & -1.975 & 0.048 \\
\hline LF & $55.1622 \pm 20.8133$ & $52.1282 \pm 10.2577$ & 0.8125 & 0.4191 \\
\hline $\mathrm{HF}$ & $44.8378 \pm 20.8133$ & $47.8718 \pm 10.2577$ & 0.8125 & 0.4191 \\
\hline Equilibrium & $0.5062 \pm 0.2447$ & $0.7203 \pm 0.1603$ & 4.5346 & $\mathrm{p}<0.001$ \\
\hline
\end{tabular}



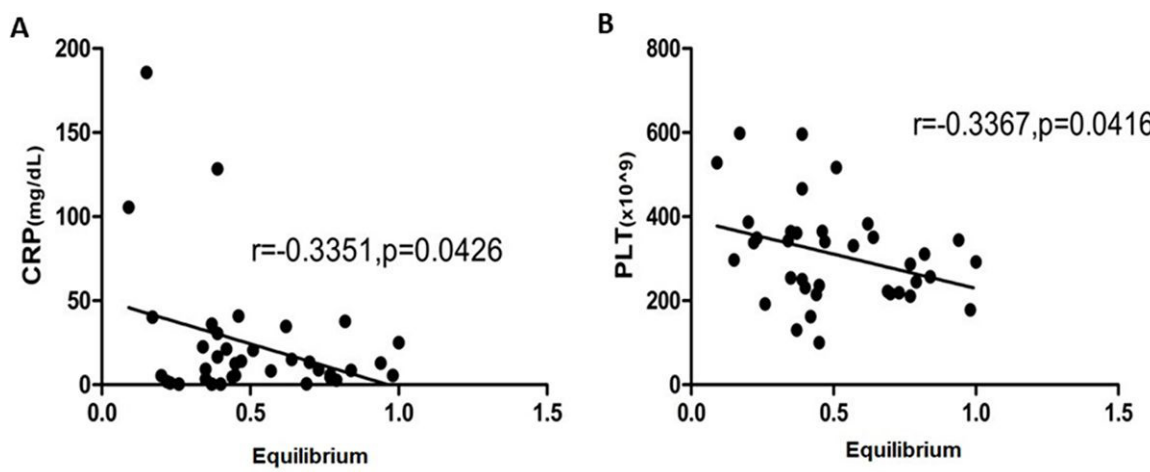

Figure 1. (A) The association between equilibrium and CRP; (B) The association between equilibrium and PLT.

Table 2. The correlation between HRV parameters and inflammatory factors, nutritional status, anxiety and depression in CD group.

\begin{tabular}{cccccc}
\hline \multirow{2}{*}{$\begin{array}{c}\text { Inflammatory } \\
\text { factors }\end{array}$} & \multicolumn{2}{c}{$\begin{array}{c}\text { Autonomic neural } \\
\text { function }\end{array}$} & & \multicolumn{2}{c}{ Equilibrium } \\
\cline { 2 - 3 } \cline { 5 - 6 } & $\mathrm{r}$ & $\mathrm{P}$ & & $\mathrm{r}$ & $\mathrm{P}$ \\
\hline WBC & -0.215 & 0.208 & & -0.24 & 0.1525 \\
CRP & -0.263 & 0.121 & & -0.3351 & 0.0426 \\
PLT & -0.141 & 0.411 & & -0.3367 & 0.0416 \\
IL-6 & -0.207 & 0.248 & & -0.2963 & 0.0888 \\
CDAI score & -0.315 & 0.061 & & -0.1251 & 0.461 \\
Nutritional status & & & & & \\
ALB & 0.210 & 0.218 & & -0.024 & 0.888 \\
BMI & 0.435 & 0.008 & & 0.3137 & 0.0587 \\
Anxiety and & & & & & \\
depression & & & & \\
SAS & -0.341 & 0.056 & & -0.3220 & 0.0676 \\
SDS & -0.294 & 0.102 & & -0.2746 & 0.1220 \\
\hline
\end{tabular}

(Sharma et al., 2009; Engel et al., 2015). It is widely accepted that the regulatory ability of autonomic nervous system declined under the condition of chronic stress or diseases.

Compared with control group, the equilibrium degree of CD group decreased significantly. The equilibrium degree reflected the balance between the sympathetic and vagus nerves, and the significantly decreased ratio reflected the dysfunction of autonomic nerve. In addition, although the difference between the groups was not statistically significant, the higher HR value indicated decreased vagus nerve tension and elevated sympathetic nerve excitability in CD group. HRV mainly reflected the equilibrium relationship between the vagus nervous system and sympathetic nervous system, and the reduction of vagus nerve tension was often used as a marker of autonomic neurofunctional disorders.

Through correlation analysis, we found that inflammatory markers such as CRP and PLT were negatively correlated with equilibrium, but had no significant association with CDAI. CRP, a common inflammatory factor, together with PLT, a factor showed high sensitivity and specificity to reflect body inflammatory state, were used to evaluate the activity level of intestinal inflammation in CD patients (Wang et al., 2008; Li et al., 2019). The negative correlation indicated that autonomic nerve equilibrium were regulated by inflammatory activities in CD patients. However, as an indicator of clinical inflammatory activity, CDAI was not fully responsive to the relationship with equilibrium, which was probably due to the influence of subjective judgment of patients.

The brain-intestinal axis theory holds that there is a wealth of autonomic nerve-forming neural clumps between the intestinal nervous system (ENS) and the central nervous system. Anxiety and depression may trigger IBD activity through inducing movement, sensation, and secretion of changes in gastrointestinal function, increasing intestinal permeability, and inhibiting the secretion of pro-inflammatory cytokines. Mittermaier et al. found that depression and anxiety symptoms were associated with early clinical recurrence of IBD and severity of the disease (Mittermaier et al., 2004). However, some studies didn't reveal the correlation between autonomic neural function and psychomental state in CD patients (Engel et al., 2015). In our study, there was no significant correlation between the HRV parameters with either anxiety or depression score in CD group, refuting the hypothesis that autonomic neurological disorders were only caused by anxiety and depression in patients with chronic or active diseases.

A number of clinical studies found the presence of autonomic nerves dysfunction in a variety of systemic autoimmune inflammatory diseases, and decreased vagus nerve tension might contribute to the pathogenesis of the disease (Evrengül et al., 2004; Wranicz et al., 2001; Gunterberg et al., 2016) However, the mechanisms underlying the causal relationship between autonomic neural dysfunction and inflammatory activity still needs to be further explored. Vagus nerve (VN), the main component of the autonomic nervous system, played an important role in the communication of brain and intestines system. ANS parasympathetic nerves could activate the HPA axis through the afferent fiber of vagus nerve, and the choline energy anti-inflammatory pathway through efferent fiber, and then inhibit inflammatory function (Tracey, 2002). In inflammatory bowel disease, vagus nerve could inhibit a variety of immune cell to produce excessive pro-inflammatory factors and suppress the process of inflammation (Bonaz \& Bernstein, 2013; Matteoli \& Boeckxstaens, 2013; Pellissier et al., 2014). In various animal models, including endotoxinemia, intestinal inflammation, and sepsis, both of electrostimulation of vagus nerve and choline neurotransmitter acetylcholine administration could inhibit the production of pro-inflammatory factor TNF-alpha, and reduce the inflammatory response (Borovikova et al., 2000; Andersson \& Tracey, 2012). In 2016, B. BONAZ first used Vagus 
Nerve Stimulation (VNS) in the treatment of patients with mild moderate $\mathrm{CD}$, and found that VNS improved autonomic nerve equilibrium and reduced inflammatory activity.(Bonaz et al., 2016) And the author speculated that VNS could strengthen the physiological anti-inflammatory pathway mediated by vagus nerve. However, the efficacy of VNS, which might be a novel direction for future $\mathrm{CD}$ diagnosis and treatment, needed to be further confirmed.

This study has several limitations. First, the sample size was small, which might influence the reliability and validity of the results. We could conduct a more detailed analysis after recruiting more participants in future study. In addition, as a retrospective study, we couldn't neglect the influence of selection bias and potential confounders. Further large-scale prospective studies were needed to confirm our findings.

Our study suggested that CD patients had decreased autonomic nerve function, mainly in equilibrium, and this change was associated with disease activity.

\section{Ethical approval}

Not applicable.

\section{Conflict of interest}

The authors declare that they have no competing interests.

\section{Funding}

This work was supported by the Science and Technology Program of Guangzhou, China (No. 202102080068).

\section{Author contributions}

WS designed the study and wrote the protocol, BW performed research/study, CC contributed important reagents, SL managed the literature searches and analyses, $\mathrm{XB}$ undertook the statistical analysis, WS wrote the first draft of the manuscript. All authors have read and approved this article.

\section{References}

Andersson, U., \& Tracey, K. J. (2012). Neural reflexes in inflammation and immunity. The Journal of Experimental Medicine, 209(6), 10571068. http://dx.doi.org/10.1084/jem.20120571. PMid:22665702.

Boissé, L., Chisholm, S. P., Lukewich, M. K., \& Lomax, A. E. (2009). Clinical and experimental evidence of sympathetic neural dysfunction during inflammatory bowel disease. Clinical and Experimental Pharmacology \& Physiology, 36(10), 1026-1033. http://dx.doi. org/10.1111/j.1440-1681.2009.05242.x. PMid:19566829.

Bonaz, B. L., \& Bernstein, C. N. (2013). Brain-gut interactions in inflammatory bowel disease. Gastroenterology, 144(1), 36-49. http:// dx.doi.org/10.1053/j.gastro.2012.10.003. PMid:23063970.

Bonaz, B., Sinniger, V., Hoffmann, D., Clarencon, D., Mathieu, N., Dantzer, C., Vercueil, L., Picq, C., Trocmé, C., Faure, P., Cracowski, J. L., \& Pellissier, S. (2016). Chronic vagus nerve stimulation in crohn's disease: a 6-month follow-up pilot study. Neurogastroenterology and Motility, 28(6), 948-953. http://dx.doi.org/10.1111/nmo.12792. PMid:26920654.
Borovikova, L. V., Ivanova, S., Zhang, M., Yang, H., Botchkina, G. I., Watkins, L. R., Wang, H., Abumrad, N., Eaton, J. W., \& Tracey, K. J. (2000). Vagus nerve stimulation attenuates the systemic inflammatory response to endotoxin. Nature, 405(6785), 458-462. http://dx.doi. org/10.1038/35013070. PMid:10839541.

Engel, T., Ben-Horin, S., \& Beer-Gabel, M. (2015). Autonomic dysfunction correlates with clinical and inflammatory activity in patients with crohn's disease. Inflammatory Bowel Diseases, 21(10), 2320-2326. PMid:26181429.

Evrengül, H., Dursunoglu, D., Cobankara, V., Polat, B., Seleci, D., Kabukcu, S., Kaftan, A., Semiz, E., \& Kilic, M. (2004). Heart rate variability in patients with rheumatoid arthritis. Rheumatology International, 24(4), 198-202. http://dx.doi.org/10.1007/s00296003-0357-5. PMid:14523570.

Ganguli, S. C., Kamath, M. V., Redmond, K., Chen, Y., Irvine, E. J., Collins, S. M., \& Tougas, G. (2007). A comparison of autonomic function in patients with inflammatory bowel disease and in healthy controls. Neurogastroenterology and Motility, 19(12), 961-967. http:// dx.doi.org/10.1111/j.1365-2982.2007.00987.x. PMid:17931336.

Group CMADMIBD. (2012). Consensus opinion on the diagnosis and treatment of inflammatory bowel disease (Guangzhou, 2012). Chinese Journal of Digestion, 32.

Gunterberg, V., Simren, M., Ohman, L., Friberg, P., Jones, M. P., Van Oudenhove, L., \& Strid, H. (2016). Autonomic nervous system function predicts the inflammatory response over three years in newly diagnosed ulcerative colitis patients. Neurogastroenterology and Motility, 28(11), 1655-1662. http://dx.doi.org/10.1111/nmo.12865. PMid:27265090.

Hill, L. K., \& Siebenbrock, A. (2009). Are all measures created equal? Heart rate variability and respiration - biomed 2009. Biomedical Sciences Instrumentation, 45, 71-76. PMid:19369742.

Kleiger, R. E., Stein, P. K., \& Bigger, J. T. Jr. (2005). Heart rate variability: measurement and clinical utility. Annals of Noninvasive Electrocardiology, 10(1), 88-101. http://dx.doi.org/10.1111/j.1542-474X.2005.10101.x. PMid:15649244.

Li, Z., Li, L., Li, X., Zhou, T., Zhang, J., \& Yang, X. (2019). Association between alb, crp and plt levels changes and mucosal healing in patients with crohn's disease. Sichuan Medicine, 40.

Malik, M. (1996). Heart rate variability: standards of measurement, physiological interpretation and clinical use. Task force of the european society of cardiology and the north american society of pacing and electrophysiology. Circulation, 93(5), 1043-1065. http:// dx.doi.org/10.1161/01.CIR.93.5.1043. PMid:8598068.

Matteoli, G., \& Boeckxstaens, G. E. (2013). The vagal innervation of the gut and immune homeostasis. Gut, 62(8), 1214-1222. http://dx.doi. org/10.1136/gutjnl-2012-302550. PMid:23023166.

Mittermaier, C., Dejaco, C., Waldhoer, T., Oefferlbauer-Ernst, A., Miehsler, W., Beier, M., Tillinger, W., Gangl, A., \& Moser, G. (2004). Impact of depressive mood on relapse in patients with inflammatory bowel disease: a prospective 18-month follow-up study. Psychosomatic Medicine, 66(1), 79-84. http://dx.doi.org/10.1097/01. PSY.0000106907.24881.F2. PMid:14747641.

Mouzas, I. A., Pallis, A. G., Kochiadakis, G. E., Marketou, M., Chlouverakis, G. I., Mellisas, J., Vardas, P. E., \& Kouroumalis, E. A. (2002). Autonomic imbalance during the day in patients with inflammatory bowel disease in remission. Evidence from spectral analysis of heart rate variability over 24 hours. Digestive and Liver Disease, 34(11), 775-780. http://dx.doi.org/10.1016/S15908658(02)80070-6. PMid:12546512.

Pellissier, S., Dantzer, C., Canini, F., Mathieu, N., \& Bonaz, B. (2010). Psychological adjustment and autonomic disturbances in inflammatory 
bowel diseases and irritable bowel syndrome. Psychoneuroendocrinology, 35(5), 653-662. http://dx.doi.org/10.1016/j.psyneuen.2009.10.004. PMid:19910123.

Pellissier, S., Dantzer, C., Mondillon, L., Trocme, C., Gauchez, A. S., Ducros, V., Mathieu, N., Toussaint, B., Fournier, A., Canini, F., \& Bonaz, B. (2014). Relationship between vagal tone, cortisol, tnf-alpha, epinephrine and negative affects in crohn's disease and irritable bowel syndrome. PLoS One, 9(9), e105328. http://dx.doi. org/10.1371/journal.pone.0105328. PMid:25207649.

Sharma, P., Makharia, G. K., Ahuja, V., Dwivedi, S. N., \& Deepak, K. K. (2009). Autonomic dysfunctions in patients with inflammatory bowel disease in clinical remission. Digestive Diseases and Sciences,
54(4), 853-861. http://dx.doi.org/10.1007/s10620-008-0424-6. PMid:18712478.

Tracey, K. J. (2002). The inflammatory reflex. Nature, 420(6917), 853-859. http://dx.doi.org/10.1038/nature01321. PMid:12490958.

Wang, B., Gao, X., Chen, M., Yang, L., Zhang, F., \& Hu, P. (2008). Changes in serum c-reactive protein levels in crohn's disease. Chinese Journal of Digestion, 28, 686-689.

Wranicz, J. K., Cygankiewicz, I., Zielinska, M., Wozniacka, A., \& SysaJedrzejowska, A. (2001). Non-invasive cardiac evaluation in patients with systemic lupus erythematosus. Journal of Medicine, 32(3-4), 195-206. PMid:11563818. 